\title{
MUSCLE DISORDERS
}

CONGENITAL INFLAMMATORY MYOPATIY

Three patients with congenital inflammatory myopathy are reported from the Montreal Children's Hospital and Neurological Institute McGill University, Montreal, Canada. Seven additional cases are reviewed from the literature. The essential criteria for diagnosis were 1) antenatal or neonatal presentation with decreased fetal movements, muscular hypotonia and weakness with or without arthrogryposis, and 2) muscle biopsy evidence for inflamatory cell infiltrate and muscle fiber damage. CPK is elevated and the EMG is myopathic. CNS involvement was present in six of the patients. Two died, one at nine months and the other at five years. Treatment with steroids was used in 7 of the 10 patients with variable results. Possible etiologies included intrauterine viral infection or an autoimmune process. (Shevell M et al. Congenital inflanmatory myopathy.

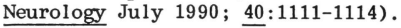

COMNENT: Congenital inflammatory myopathy is a rare cause of the "floppy infant syndrome", and the differential diagnosis includes a congenital muscular dystrophy syndrome of Fukuyama or Walker-Warburg. Fukuyama's congenital muscular dystrophy includes hydrocephalus, ocular manifestations and mental retardation and Walker-Warburg's syndrome is characterized by lissencephaly and retinal abnormalities. Treatment of the cases of congenital inflammatory myopathy with steroids may improve motor development but does not result in intellectual improvement.

\section{EARLY DEVELOPMENT IN DUCHENNE MUSCULAR DYSTROPHY}

The early development of 33 boys wi th Duchenne muscular dystrophy

(IMD) was assessed at the Institute of Miedical Genetics, University of Wales College of Medicine, Heath Park, Cardiff, Wales. The average age of entry into the study was 3.4 years (range 0.8 to 6.7 ). The Griffiths Developmental Scales, The Reynell Language Scales and the British Picture Vocabulary Scales were used at three evaluations at 6 monthly intervals over a one year period. Compared to a control group the boys with DMD showed developmental delay which was most severe in the locomotor and language areas. Locomotor quotients deteriorated over time and behavior problems were probably secondary to the developmental delay. (Smith RA et al Early development of boys with Duchenne muscular dystrophy. Dev Med Child Neurol June 1990; 32:519-527).

COMNENT: Unexplained developmental delays should prompt a neurological evaluation to exclude Duchenne muscular dystrophy in boys. Young children wi th $\mathrm{DMD}$ have significant problems with motor, speech and behavioral disorders before muscle weakness becomes an obvious clinical sign.

The etiology of intellectual impairment in Duchenne muscular dystrophy was investigated by MRI studies at Jordan University, 
Amman, Jordan (Al-Qudah AA et al. Pediatr Neurol 1990; 6:57-59). Four $\mathrm{DMD}$ patients were studied prospectively by cranial $\mathrm{MRI}$, DNA deletion analysis, clinical evaluation and IQ testing. There was no significant correlation between verbal IQ and MRI findings, DNA deletion or the clinical severity of the disease. Other than mild atrophy in two patients, no significant anatamical brain lesion was discovered. Previous reports have attributed intellectual impairment in $\mathrm{MM}$ to anatomic brain changes, abnormal dendritic development, and migrational lesions. Others have described a direct relation between the severity of clinical disease and impairment of IQ. (Rosman NP Brain 1966 $89: 769)$.

\section{GENETICS OF DUCHENNE AND BECKER DYSTROPHIES}

The molecular basis for Duchenne and Becker muscular dystrophies is reviewed from the Department of Pediatric Neurology, Floating Hospital for Infants and Children, New England Medical Center Hospitals and Tufts University, Boston, Mass. Until recently the diagnosis of $\mathrm{DMD}$ or BMD depended on clinical signs and symptoms, serum creatine kinase, EMG and muscle biopsy. The isolation of the gene defective in $\mathrm{DMD}$ and $\mathrm{BMD}$ and the identification of dystrophin have revolutionized the diagnostic issues. The mutated gene causing Duchenne and its allelic milder Becker phenotype has been assigned to band P21 of the short arm of the $\mathrm{X}$ chromosame (Xp21). Dystrophin has been characterized by DNA sequencing and by immunologic studies. When the family history is negative for DMD and BMD the Western Blot assay of protein derived from a specimen can confirm the clinical diagnosis of DMD or BMD and can be used to predict the severity of the disease. If the dystrophin assay result is abnormal DNA analysis should be performed. Detection of a dystrophin gene deletion will facilitate carrier detection and prenatal diagnosis in the proband's family. If no deletion or duplication is found, linkage analysis may be attempted for prenatal diagnosis and carrier detection. Peripheral blood DNA may be used for Southern Blot testing if a muscle biopsy specimen is not available and may confirm the diagnosis in more than $65 \%$ of the cases. In typical cases of IND or BMD with a family history of $\mathrm{X}$ linked muscular dystrophy, linkage analysis is unnecessary if the clinical diagnosis has been confirmed in an affected family member by analysis of dystrophin or DNA or both. The less invasive polymerase chain reaction test may be used if the diagnosis has not been confirmed by dystrophin or DNA analysis in other family members. Muscle biopsy for dystrophin analysis will be required in families without a clear cut $X$ linked pattern of inheritance or in families with both male and female siblings affected, suggesting an autosomal recessive form of $M$ (Darras BT. Molecular Genetics of Duchenne and Becker muscular dystrophy. J Pediat July $1990 ; 117: 1-15)$.

COMMENT: Gross abnormalities of the dystrophin gene may still result in a partially functional dystrophin protein and a relatively mild clinical progression, compatible with a diagnosis of Becker muscular dystrophy. Angelini C et a. (Neurology May 